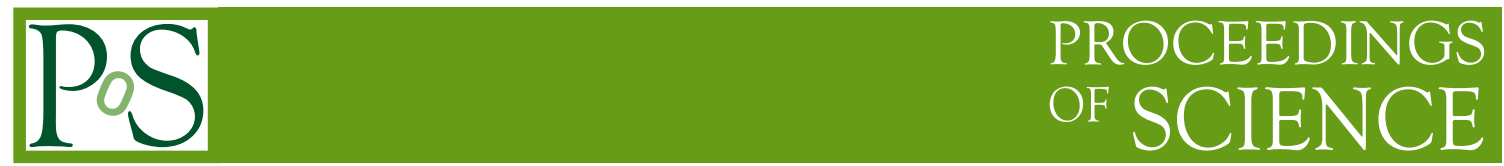

\title{
Gamma-ray properties of low luminosity AGNs
}

\author{
Rafal Wojaczynski* \\ University of Lodz, Department of Astrophysics \\ E-mail: rafal.wojaczynski@wp.pl
}

\begin{abstract}
We present results of our analysis of the Fermi-LAT data from low-luminosity Seyfert galaxies. We find a significant $\gamma$-ray detection in NGC 6814, marginally significant $(\sim 3 \sigma)$ signals at the positions of NGC 4151 and NGC 4258 and no $\gamma$-ray signals at the positions of other objects. We then derive upper limits for the $\gamma$-ray flux. We find that by comparing them with the $\gamma$-ray fluxes predicted by the hot flow model, which explains the X-ray spectra of the considered objects, we can constrain the fraction of the accretion power used for relativistic acceleration of protons to at most $\sim 10 \%$. In some, the LAT upper limits also allow to constrain the magnetic field strength and black hole spin values.
\end{abstract}

The 34th International Cosmic Ray Conference,

30 July- 6 August, 2015

The Hague, The Netherlands

\footnotetext{
* Speaker.
} 


\section{Introduction}

Low-luminosity AGNs are likely to be powered by optically thin, hot accretion flows. In central parts of such flows, protons have energies above the threshold for pion production and then the flows produce considerable $\gamma$-ray fluxes due to the decay of neutral pions. Below we refer to predictions for such a hadronic $\gamma$-ray emission obtained with the precise model of [1,2], which is fully general relativistic and takes into account absorption of $\gamma$-rays in the radiation field of the flow.

On the observational ground, no prominent $\gamma$-ray signal was found in the Fermi/LAT data from radio-quiet AGNs, e.g. [3,4], with a few notable exceptions. These include the three X-ray brightest Seyfert 2 galaxies, NGC 4945, NGC 1068 and Circinus which are, however, composite AGN/starburst systems and it has not yet been definitively established whether their $\gamma$-ray emission is related with the starburst or AGN activity. The upper limits (UL) on the photon flux in the GeV range, derived in [3], correspond to the $\gamma$-ray to X-ray luminosity ratio values comparable with those predicted by the hot flow model. This shows that sensitivity of the Fermi/LAT surveys has reached the level at which predictions of the hadronic emission from hot accretion flows can be probed and motivates us to make the detailed comparison reported below. Details of our analysis and results are presented in [5].

\section{Data analysis}

We analyzed Fermi/LAT data from several nearby Seyfert 1 galaxies: NGC 4151, NGC 4258, NGC 5548, IC 4329a, NGC 6814, and NGC 7213. Analysis was done using Fermi Science Tools package delivered by HEASARC at the Goddard Space Flight Center. At the time of analysis we used all possible data which resulted in processing dataset of 6.4 years, comprising observations carried out between 2008 August 4 and 2015 January 10. We used CALDB response function and analysis type used was unbinned likelihood analysis. For each object events were selected with radius $10^{\circ}$ around the analyzed source. Models built for each object consist of all gamma-ray sources from 3 FGL catalogue [4] in radius $15^{\circ}$ around the source. We used the standard templates for the Galactic (g11_iem_v05_rev1.fits) and the isotropic (iso_source_v05_rev1.txt) backgrounds. All of the sources were modeled using power-law spectral function with parameters left free during gtlike fitting. Except for NGC 6814 we do not find statistically significant signals and then we derive the $95 \%$ confidence level ULs for the photon flux, $F$, using the Bayesian method. ULs were calculated in two energy ranges $1-10 \mathrm{GeV}$ and $0.2-1 \mathrm{GeV}$, which allow to compare with predictions for the nonthermal and thermal distribution of protons, respectively. Our results are summarized in Table $\mathbb{\square}$.

\subsection{NGC 6814}

This object revealed the highest value of TS $=32.2$ among all Seyfert 1 galaxies. Our TS values, photon flux and the fitted power-law index are consistent with those found in [3] using 3 years of LAT data. It is not clear whether this signal represents an actual emission from NGC 6814 or it is due to a spatial coincidence with another source. In the former case, it cannot be a direct $\gamma$-ray emission from a hot flow at its current level of the X-ray luminosity. On the other hand, it can 
Table 1: Integrated photon flux, $F$, and the photon index, $\Gamma$, of power-law fits, or $95 \%$ confidence level ULs with the assumed $\Gamma$, and the corresponding Eddington ratio for 1-10 GeV (upper part) and 0.2-1 GeV (lower part). ${ }^{(a)}$ using full data set for 6.4 years and including source $S$ in the model; ${ }^{(b)} \sim 4.9$-years data set $(6.4$ years with excluded period S), source S not included; ${ }^{(c)}$ data from first 3 years, S included in the model; ${ }^{\text {(d) }}$ data from first 3 years, S not included in the model.

\begin{tabular}{|c|c|c|c|}
\hline Source & $\Gamma$ & $\begin{array}{c}F \\
10^{-10} \mathrm{ph} / \mathrm{cm}^{2} / \mathrm{s}\end{array}$ & $\begin{array}{c}L_{\gamma} / L_{\text {Edd }} \\
\times 10^{-5}\end{array}$ \\
\hline \multicolumn{4}{|c|}{ Energy range: $1-10 \mathrm{GeV}$} \\
\hline IC $4329 a$ & 2.7 & $<0.46$ & $<0.49$ \\
\hline IC 4329a & 2.1 & $<0.68$ & $<0.88$ \\
\hline NGC 4151 (a) & 2.7 & $<1.2$ & $<0.23$ \\
\hline NGC $4151^{\text {(a) }}$ & 2.1 & $<1.3$ & $<0.31$ \\
\hline NGC $4151^{(b)}$ & 2.1 & $<1.8$ & $<0.43$ \\
\hline NGC 4151 (c) & 2.1 & $<1.8$ & $<0.43$ \\
\hline NGC $4151^{(d)}$ & 2.1 & $<2.0$ & $<0.48$ \\
\hline NGC 4258 & 2.7 & $<1.1$ & $<0.043$ \\
\hline NGC 4258 & 2.1 & $<1.5$ & $<0.067$ \\
\hline NGC 5548 & 2.1 & $<0.3$ & $<1.8$ \\
\hline NGC 5548 & 2.7 & $<0.6$ & $<2.9$ \\
\hline NGC 6814 & $2.6 \pm 0.1$ & $3.0 \pm 0.7$ & $18 \pm 10$ \\
\hline NGC 6814* & $2.6 \pm 0.1$ & $3.0 \pm 0.7$ & $2.6 \pm 1$ \\
\hline NGC 7213 & 2.7 & $<0.4$ & $<0.07$ \\
\hline NGC 7213 & 2.1 & $<0.5$ & $<0.11$ \\
\hline \multicolumn{4}{|c|}{ Energy range: $0.2-1 \mathrm{GeV}$} \\
\hline IC 4329a & 4.0 & $<3.3$ & $<0.51$ \\
\hline NGC $4151^{\text {(a) }}$ & 4.0 & $<4.5$ & $<0.13$ \\
\hline NGC $4151^{(b)}$ & 4.0 & $<20$ & $<0.52$ \\
\hline NGC 4258 & 4.0 & $<7.7$ & $<0.044$ \\
\hline NGC 5548 & 4.0 & $<12$ & $<8.4$ \\
\hline NGC 7213 & 4.0 & $<7.1$ & $<0.18$ \\
\hline
\end{tabular}

result from hadronic emission of protons injected from the active nucleus into the circumnuclear matter in the tentative past period of higher activity; in such a scenario, NGC 6814 would present some similarities to the $\gamma$-ray loud Seyfert 2 galaxies, see [5].

\subsection{NGC 4258}

NGC 4258 has not been included in previous analyzes of the Fermi/LAT data. At its position we find a weak $\gamma$-ray excess above the background, with TS $=9.4$. NGC 4258 has a low $2-10 \mathrm{keV}$ luminosity, $\simeq 10^{-5} L_{\text {Edd }}$, at which the $0.2-1 \mathrm{GeV}$ emission is weakly affected by $\gamma \gamma$ absorption and, if this galaxy contains a rapidly rotating supermassive black hole, we can directly receive $\gamma$-rays from its ergospheric region. However, even for the maximum spin value, the expected signal is only at the level of the Fermi-LAT UL for $0.2-1 \mathrm{GeV}$. The UL derived for $1-10 \mathrm{GeV}$ is below the flux 
predicted from a hot flow containing a significant content of nonthermal protons, which constrains the acceleration efficiency of protons to at most $\sim 10 \%$.

\subsection{NGC 7213}

NGC 7213 is observed at the $2-10 \mathrm{keV}$ luminosity of $\simeq 10^{-4} L_{\text {Edd }}$ and then it gives interesting constraints on the hot flow model at moderate Eddington ratios, between those of the X-ray bright Seyfert 1 galaxies, like NGC 4151, and the weakly luminous NGC 4258. Our likelihood analysis of the Fermi/LAT data does not reveal any significant signal around NGC 7213 (TS < 1). The UL derived for 1-10 GeV energy range constrains the nonthermal energy content in the proton distribution to $\sim 10 \%$, similarly as for NGC 4258. Furthermore, for this AGN the UL for $0.2-1$ $\mathrm{GeV}$ energy range rules out a weakly magnetized flow around a rapidly rotating black hole.

\subsection{NGC 4151}

At the position of NGC 4151 we find a signal with the test-statistic significance TS $\simeq 17$ for $E=0.2-6 \mathrm{GeV}$, see Fig. Шd. It likely contains a contribution from a new $\gamma$-ray source found in our analysis, denoted below by $\mathrm{S}$, shifted by only $\sim 0.5$ degrees from NGC 4151 . Interestingly, however, the gtfindsrc best-fit location for this signal (shown by the black cross), assuming that it comes from a single source, is shifted from the position of NGC 4151 by only 8 arcminutes. Source $\mathrm{S}$ is strongly variable, which hinders a proper assessment of its contribution to the $\gamma$-ray signal. Its position is determined by several photons with energies between 10 and $20 \mathrm{GeV}$, which arrived from the same direction (within $\sim 8$ arcminutes) between December 2011 and June 2013; we assume that the source exhibited an outburst activity during this 1.5 -year period, which we denote as period S. Indeed, the source S is clearly seen in the TS map built for period S only, see Fig. Пc, whereas the map built by neglecting this period shows only a weak residual, centered on NGC 4151 rather than S, see Fig. Wa. After subtracting all 3FGL sources, we find for source S: (1) $\mathrm{TS} \simeq 30, \Gamma=1.78 \pm 0.37, F_{>0.1 \mathrm{GeV}} \simeq 1.2 \times 10^{-9} \mathrm{ph} \mathrm{s}^{-1} \mathrm{~cm}^{-2}$ using the data from period $\mathrm{S}$, and (2) $\mathrm{TS} \simeq 22, \Gamma=2.17 \pm 0.20, F_{>0.1 \mathrm{GeV}} \simeq 2.1 \times 10^{-9} \mathrm{ph} \mathrm{s}^{-1} \mathrm{~cm}^{-2}$ using the full 6.4-years data set. Source $\mathrm{S}$ is not reported in 3FGL, which was built using data up to 2012 July, i.e. covering only $~ 30 \%$ of period S. For the data from period S, gtfindsrc gives the location of $\mathrm{S}$ at $\alpha_{J 2000}=12^{\mathrm{h}} 11^{\mathrm{m}} 27^{\mathrm{s}}$, $\delta_{J 2000}=38^{\circ} 56^{\prime} 48^{\prime \prime}$. A possible candidate for this source is a BL Lac object, 2E 1209.0+3917, only $\simeq 4$ arcminutes from this location. For a 4.9 -year data set, with neglected period S, gtlike shows no signal ( $\mathrm{TS} \simeq 1.5$ ) at the $\mathrm{S}$ position. The residual seen in the TS map for this data set, Fig. W(a), can be fully compensated for by adding the point source at the position of NGC 4151 (compare with panel b) and gtlike gives $T S \simeq 8, \Gamma=2.7 \pm 0.3$ and $F_{>0.1 \mathrm{GeV}}=(1.5 \pm 0.6) \times 10^{-9}$ for such a source.

NGC 4151 is a bright Seyfert 1 , with the intrinsic $2-10 \mathrm{keV}$ luminosity of $\simeq 10^{-3} L_{\text {Edd. }}$. The $1-10 \mathrm{GeV}$ UL constrains the energy content of a nonthermal proton component to $\sim 10 \%$, similarly as in Seyfert galaxies with lower X-ray luminosities. The $0.2-1 \mathrm{GeV}$ UL disfavors models with both a large black hole spin and a weak magnetic field, in a manner consistent with NGC 7213, however, this result is model-dependent. Namely, the presence of a variable source $\mathrm{S}$ results in an ambiguity for the UL on the $\gamma$-ray flux from NGC 4151 below $1 \mathrm{GeV}$, where the point spread function of LAT is much larger than the angular separation between S and NGC 4151. 


\subsection{NGC 5548, IC 4329a}

These two Seyfert 1 galaxies are typically observed at $L_{2-10 \mathrm{keV}}$ exceeding $10^{-3} L_{\mathrm{Edd}}$, which is the maximum luminosity which can be studied with the precise model of [1]. Both show no $\gamma$-ray signal, with TS $<1$. This shows that low $\gamma$-ray activity of Seyfert galaxies sustains in the regime of an efficient Coulomb cooling of protons, which should lead to the increase of density and hence the increase of proton-proton interaction rate.

\section{Summary}

Observations in the $\mathrm{GeV}$ range provide the means to directly measure the efficiency of proton acceleration in hot flows. We explored the $\gamma$-ray activity of several nearby Seyfert galaxies, for which the available data allow a robust determination of the nuclear luminosity scaled by the Eddington value, which is crucial for comparison with the hot flow model predictions. The obtained ULs for the 1-10 GeV flux in NGC 4258, NGC 7213 and NGC 4151 constrain the fraction of accretion power used for relativistic acceleration of protons to at most $10 \%$. Remarkably, these three AGNs allow to probe various parts of the flow, because at $L_{2-10 \mathrm{keV}} \sim 10^{-5}, 10^{-4}$ and $10^{-3} L_{\mathrm{Edd}}$, radiation produced at a few, ten and several tens of $R_{\mathrm{g}}$, respectively, dominates the observed 1-10 GeV flux.

\section{Acknowledgments}

This work was supported in part by the Polish MNiSW grant for young scientists B1511500001045.02.

\section{References}

[1] Niedźwiecki, A., Xie, F.-G., \& Stępnik, A. 2013, MNRAS, 432, 1576

[2] Niedźwiecki A., Stępnik A. \& Xie F.-G. 2015, ApJ, 799, 217

[3] Ackermann, M., Ajello, M., Allafort, A., et al. 2012, ApJ, 755, 164

[4] The Fermi-LAT Collaboration. 2015, ArXiv e-prints [arXiv:1501.02003]

[5] Wojaczynski, R., Niedźwiecki, A., Xie, F.-G., Szanecki, M., 2015, ArXiv e-prints[arXiv:1505.07608] 

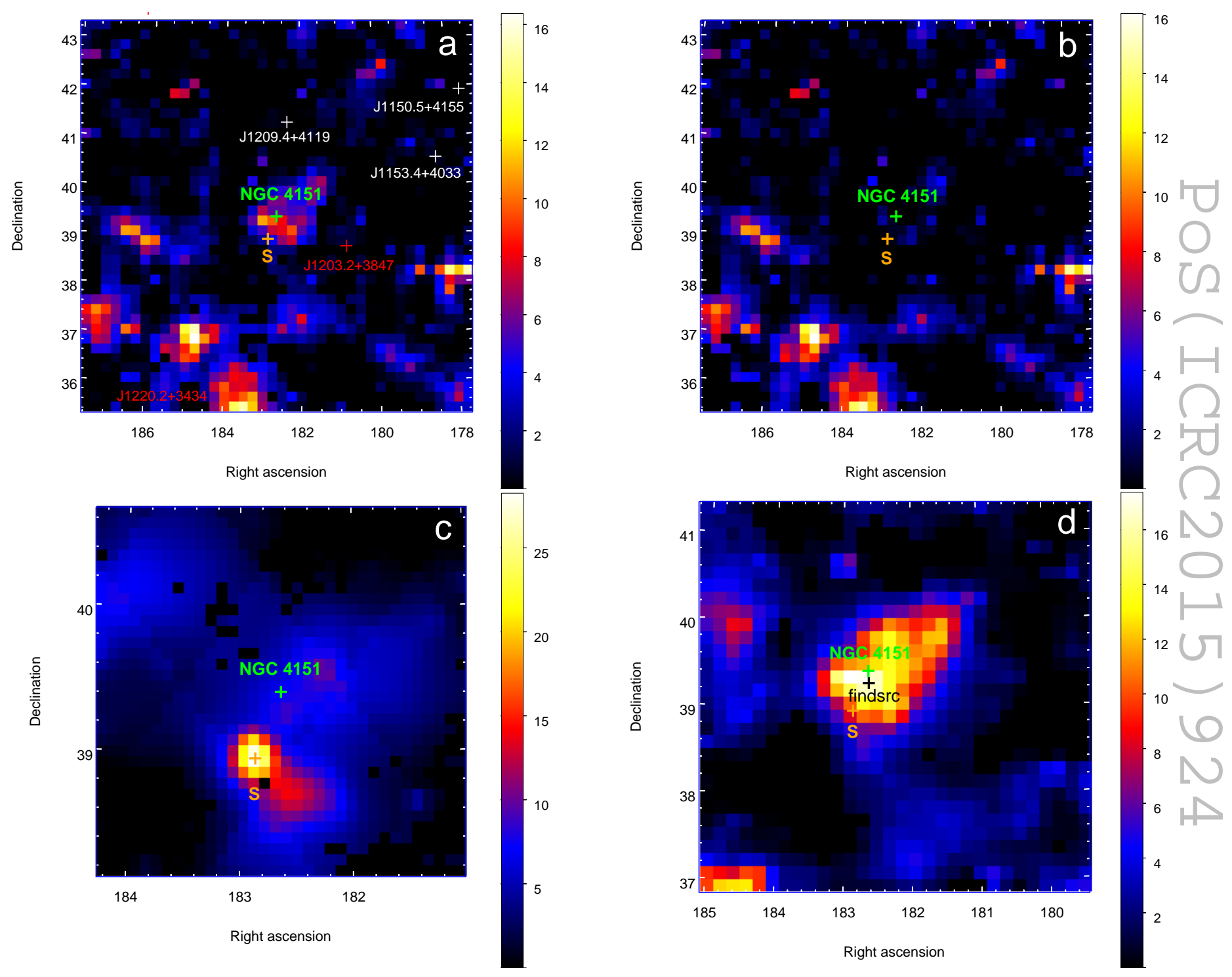

Figure 1: Test statistics maps showing region near NGC 4151. It's position is marked as green cross, source $\mathrm{S}$ marked as orange on all panels. On panel (a) position of 5 available sources in 3FGL catalogue marked as white (available in 2FGL) and red (added in 3FGL). Area size: $8^{\circ} \times 8^{\circ}$, energy range: $0.2-30 \mathrm{GeV}$, period 4.9 year neglecting period S. (b) The same energy and period as panel (a), added point source at position NGC 4151. (c) Area size: $2.55^{\circ} \times 2.55^{\circ}$, energy range $0.2-30 \mathrm{GeV}$, period 1.5 year $=\mathrm{S}$ (d) Area size: $4.8^{\circ} \times 4.8^{\circ}$, energy range $0.2-6 \mathrm{GeV}$, full period 6.4 years. Source $\mathrm{S}$ not visible due to neglect photons $>$ $10 \mathrm{GeV}$, black cross shows position of source find by findsrc. 